\title{
On Bayesian Estimation of Densities and SAmpling Distributions: the Posterior Predictive Distribution as the BAYES Estimator
}

\author{
A.G. Nogales \\ Dpto. de Matemáticas, Universidad de Extremadura \\ Avda. de Elvas, s/n, 06006-Badajoz, SPAIN. \\ e-mail: nogales@unex.es
}

\begin{abstract}
Optimality results for two outstanding Bayesian estimation problems are given in this paper: the estimation of the sampling distribution for the squared total variation function and the estimation of the density for the $L^{1}$-squared loss function. The posterior predictive distribution provides the solution to these problems. Some examples are presented to illustrate it. The Bayesian estimation problem of a distribution function is also addressed. Consistency of the estimator of the density is proved.
\end{abstract}

AMS Subject Class. (2020): Primary 62F15, 62G07 Secondary 62C10

Key words and phrases: Bayesian density estimation, posterior predictive distribution. 


\section{Introduction and basic definitions}

The posterior predictive distribution has been introduced in the literature to predict the distribution of a future observation from the previous random sample (see Gelman et al. (2014) or Boldstat (2004)), but it has also been used in other areas such as model selection, testing for discordancy, goodness of fit, perturbation analysis or classification. Other fields of application are presented in Geisser (1993) and Rubin (1984).

In the next pages, the problems of estimation of a density or a probability measure is considered under the Bayesian point of view. These problems are addressed in a number of previous books and papers such as Ghosh et al. (2003, Ch. 5), Lijoi et al. (2010, sect. 3.4), Lo (1984) or Ferguson (1983), to mention just a few. Ghosal et al. (2017), p. 121, contains a brief historical review on Bayesian density estimation. Here, the posterior predictive density appears as the optimal estimator of the density for the $L^{1}$-squared loss function and an arbitrary prior distribution. In fact, the posterior predictive distribution is the optimal estimator of the probability measures $P_{\theta}$ for the squared total variation loss function. These results come to endorse the applications of the posterior predictive distribution above mentioned.

Several examples are presented in Section 4 to illustrate the results. Gelman et al. (2014) contains many other examples (and exercises) of determination of the posterior predictive distribution. But in practice, the explicit evaluation of the posterior predictive distribution could be cumbersome and its simulation may become preferable. Gelman et al. (2014) is a good reference also for such simulation methods.

In what follows we will place ourselves in a general framewok for the Bayesian inference, as is described in Barra (1971), for instance, covering simultaneously the discrete, continuous, univariate and multivariate cases.

First, let us briefly recall some basic concepts about Markov kernels. In the next, $(\Omega, \mathcal{A}),\left(\Omega_{1}, \mathcal{A}_{1}\right)$ and so on will denote measurable spaces.

Definition 1. 1) (Markov kernel) A Markov kernel $M_{1}:(\Omega, \mathcal{A}) \succ\left(\Omega_{1}, \mathcal{A}_{1}\right)$ is a map $M_{1}: \Omega \times \mathcal{A}_{1} \rightarrow[0,1]$ such that: (i) $\forall \omega \in \Omega, M_{1}(\omega, \cdot)$ is a probability measure on $\mathcal{A}_{1}$; (ii) $\forall A_{1} \in \mathcal{A}_{1}, M_{1}\left(\cdot, A_{1}\right)$ is $\mathcal{A}$-measurable.

2) (Image of a Markov kernel) The image (or probability distribution) of a Markov kernel $M_{1}:(\Omega, \mathcal{A}, P) \succ\left(\Omega_{1}, \mathcal{A}_{1}\right)$ on a probability space is the probability measure $P^{M_{1}}$ on $\mathcal{A}_{1}$ defined by $P^{M_{1}}\left(A_{1}\right):=\int_{\Omega} M_{1}\left(\omega, A_{1}\right) d P(\omega)$.

3) (Composition of Markov kernels) Given two Markov kernels $M_{1}$ : $\left(\Omega_{1}, \mathcal{A}_{1}\right) \succ\left(\Omega_{2}, \mathcal{A}_{2}\right)$ and $M_{2}:\left(\Omega_{2}, \mathcal{A}_{2}\right) \succ\left(\Omega_{3}, \mathcal{A}_{3}\right)$, its composition is de- 
fined as the Markov kernel $M_{2} M_{1}:\left(\Omega_{1}, \mathcal{A}_{1}\right) \succ\left(\Omega_{3}, \mathcal{A}_{3}\right)$ given by

$$
M_{2} M_{1}\left(\omega_{1}, A_{3}\right)=\int_{\Omega_{2}} M_{2}\left(\omega_{2}, A_{3}\right) M_{1}\left(\omega_{1}, d \omega_{2}\right)
$$

Remarks. 1) (Markov kernels as extensions of the concept of random variable) The concept of Markov kernel extends the concept of random variable (or measurable map). A random variable $T_{1}:(\Omega, \mathcal{A}, P) \rightarrow\left(\Omega_{1}, \mathcal{A}_{1}\right)$ will be identified with the Markov kernel $M_{T_{1}}:(\Omega, \mathcal{A}, P) \succ\left(\Omega_{1}, \mathcal{A}_{1}\right)$ defined by $M_{T_{1}}\left(\omega, A_{1}\right)=\delta_{T_{1}(\omega)}\left(A_{1}\right)=I_{A_{1}}\left(T_{1}(\omega)\right)$, where $\delta_{T_{1}(\omega)}$ denotes the Dirac measure -the degenerate distribution- at the point $T_{1}(\omega)$, and $I_{A_{1}}$ is the indicator function of the event $A_{1}$. In particular, the probability distribution $P^{M_{T_{1}}}$ of $M_{T_{1}}$ coincides with the probability distribution $P^{T_{1}}$ of $T_{1}$ defined as $P^{T_{1}}\left(A_{1}\right):=P\left(T_{1} \in A_{1}\right)$

2) Given a Markov kernel $M_{1}:\left(\Omega_{1}, \mathcal{A}_{1}\right) \succ\left(\Omega_{2}, \mathcal{A}_{2}\right)$ and a random variable $X_{2}:\left(\Omega_{2}, \mathcal{A}_{2}\right) \rightarrow\left(\Omega_{3}, \mathcal{A}_{3}\right)$, we have that $M_{X_{2}} M_{1}\left(\omega_{1}, A_{3}\right)=M_{1}\left(\omega_{1}, X_{2}^{-1}\left(A_{3}\right)\right)=$ $M_{1}\left(\omega_{1}, \cdot\right)^{X_{2}}\left(A_{3}\right)$. We write $X_{2} M_{1}:=M_{X_{2}} M_{1}$.

Let $\left(\Omega, \mathcal{A},\left\{P_{\theta}: \theta \in(\Theta, \mathcal{T}, Q)\right\}\right)$ be a Bayesian statistical experiment where $Q$ is the prior distribution, a probability measure on the measurable space $(\Theta, \mathcal{T}) .(\Omega, \mathcal{A})$ is the sample space and $(\Theta, \mathcal{T})$ is the parameter space.

When needed, we shall suppose that $P_{\theta}$ has a density (or Radon-Nikodym derivative) $p_{\theta}$ with respect to a $\sigma$-finite measure $\mu$ on $\mathcal{A}$ and that the likelihood function $\mathcal{L}:(\omega, \theta) \in(\Omega \times \Theta, \mathcal{A} \otimes \mathcal{T}) \rightarrow \mathcal{L}(\omega, \theta):=p_{\theta}(\omega)$ is measurable. So we have a Markov kernel $P:(\Theta, \mathcal{T}) \succ(\Omega, \mathcal{A})$ defined by $P(\theta, A):=P_{\theta}(A)$. Let $P^{*}:(\Omega, \mathcal{A}) \succ(\Theta, \mathcal{T})$ the Markov kernel determined by the posterior distributions. In fact, if we denote by $\Pi$ the only probability measure on $\mathcal{A} \otimes \mathcal{T}$ such that

$$
\Pi(A \times T)=\int_{T} P_{\theta}(A) d Q(\theta), \quad A \in \mathcal{A}, T \in \mathcal{T},
$$

then $P^{*}$ is defined in such a way that

$$
\Pi(A \times T)=\int_{A} P_{\omega}^{*}(T) d \beta_{Q}^{*}(\omega), \quad A \in \mathcal{A}, T \in \mathcal{T},
$$

where $\beta_{Q}^{*}$ denotes the so called prior predictive probability, defined by

$$
\beta_{Q}^{*}(A)=\int_{\Theta} P_{\theta}(A) d Q(\theta), \quad A \in \mathcal{A} .
$$

In other terms, $\beta_{Q}^{*}=Q^{P}$, the probability distribution of the Markov kernel $P$ with respect to the prior distribution $Q$. 
The probability measure $\Pi$ integrates all the basic ingredients of the Bayesian model, and these ingredients can be essentially derived from $\Pi$, something that would allow us to identify the Bayesian model as the probability space $(\Omega \times \Theta, \mathcal{A} \otimes \mathcal{T}, \Pi)$ (so is done, for instance, in Florens et al. (1990)).

It is well known that, for $\omega \in \Omega$, the posterior density with respect to the prior distribution is proportional to the likelihood. Namely

$$
p_{\omega}^{*}(\theta):=\frac{d P_{\omega}^{*}}{d Q}(\theta)=C(\omega) p_{\theta}(\omega)
$$

where $C(\omega)=\left[\int_{\Theta} p_{\theta}(\omega) d Q(\theta)\right]^{-1}$.

This way we obtain a statistical experiment $\left(\Theta, \mathcal{T},\left\{P_{\omega}^{*}: \omega \in \Omega\right\}\right)$ on the parameter space $(\Theta, \mathcal{T})$. We can reconsider the Markov kernel $P$ defined on this statistical experiment

$$
P:\left(\Theta, \mathcal{T},\left\{P_{\omega}^{*}: \omega \in \Omega\right\}\right) \succ(\Omega, \mathcal{A}) .
$$

Since $\left(P_{\omega}^{*}\right)^{P}(A)=\int_{\Theta} P_{\theta}(A) d P_{\omega}^{*}(\theta)$, for $A \in \mathcal{A}$, it is called the posterior predictive distribution on $\mathcal{A}$ given $\omega$, and the statistical experiment image of $P$ is

$$
\left(\Omega, \mathcal{A},\left\{\left(P_{\omega}^{*}\right)^{P}: \omega \in \Omega\right\}\right) .
$$

Note that, given $\omega \in \Omega$, according to Fubini's Theorem,

$$
\begin{aligned}
\left(P_{\omega}^{*}\right)^{P}(A) & =\int_{\Theta} P_{\theta}(A) d P_{\omega}^{*}(\theta)=\int_{\Theta} \int_{A} p_{\theta}\left(\omega^{\prime}\right) d \mu\left(\omega^{\prime}\right) p_{\omega}^{*}(\theta) d Q(\theta) \\
& =\int_{A} \int_{\Theta} p_{\theta}\left(\omega^{\prime}\right) p_{\omega}^{*}(\theta) d Q(\theta) d \mu\left(\omega^{\prime}\right) .
\end{aligned}
$$

So, the posterior predictive density is

$$
\frac{d\left(P_{\omega}^{*}\right)^{P}}{d \mu}\left(\omega^{\prime}\right)=\int_{\Theta} p_{\theta}\left(\omega^{\prime}\right) p_{\omega}^{*}(\theta) d Q(\theta)
$$

If we consider the composition of the Markov kernels $P^{*}$ and $P$ :

$$
(\Omega, \mathcal{A}) \stackrel{P^{*}}{\longrightarrow}(\Theta, \mathcal{T}) \stackrel{P}{\longrightarrow}(\Omega, \mathcal{A}),
$$

defined by

$$
P P^{*}(\omega, A):=\int_{\Theta} P_{\theta}(A) d P_{\omega}^{*}(\theta)=\int_{A} \int_{\Theta} p_{\theta}\left(\omega^{\prime}\right) p_{\omega}^{*}(\theta) d Q(\theta) d \mu\left(\omega^{\prime}\right),
$$


we have that

$$
\frac{d P P^{*}(\omega, \cdot)}{d \mu}\left(\omega^{\prime}\right)=\int_{\Theta} p_{\theta}\left(\omega^{\prime}\right) p_{\omega}^{*}(\theta) d Q(\theta) .
$$

Notice that $P P^{*}(\omega, \cdot)=\left(P_{\omega}^{*}\right)^{P}$.

Remarks. 1) Analogously, if we consider the Markov kernel

$$
P^{*}:\left(\Omega, \mathcal{A},\left\{P_{\theta}: \theta \in \Theta\right\}\right) \succ\left(\Theta, \mathcal{T},\left\{\left(P_{\theta}\right)^{P^{*}}: \theta \in \Theta\right\}\right),
$$

we have that

$$
\left(P_{\theta}\right)^{P^{*}}(T)=\int_{\Omega} P_{\omega}^{*}(T) d P_{\theta}(\omega)=\int_{T} \int_{\Omega} p_{\omega}^{*}\left(\theta^{\prime}\right) p_{\theta}(\omega) d \mu(\omega) d Q\left(\theta^{\prime}\right)
$$

and

$$
\frac{d\left(P_{\theta}\right)^{P^{*}}}{d Q}\left(\theta^{\prime}\right)=\int_{\Omega} p_{\omega}^{*}\left(\theta^{\prime}\right) p_{\theta}(\omega) d \mu(\omega) .
$$

Notice that $\left(\beta_{Q}^{*}\right)^{P^{*}}(T)=\int_{\Omega} P_{\omega}^{*}(T) d \beta_{Q}^{*}(\omega)=Q(T)$, i.e., $\left(\beta_{Q}^{*}\right)^{P^{*}}=Q$.

If we consider the composition of the Markov kernels $P$ and $P^{*}$ :

$$
(\Theta, \mathcal{T}) \stackrel{P}{\longrightarrow}(\Omega, \mathcal{A}) \stackrel{P^{*}}{\longrightarrow}(\Theta, \mathcal{T}),
$$

defined by

$$
P^{*} P(\theta, T)=\int_{\Omega} P_{\omega}^{*}(T) d P_{\theta}(\omega)=\int_{T} \int_{\Omega} p_{\omega}^{*}\left(\theta^{\prime}\right) p_{\theta}(\omega) d \mu(\omega) d Q\left(\theta^{\prime}\right),
$$

we obtain

$$
\frac{d P^{*} P(\theta, \cdot)}{d Q}\left(\theta^{\prime}\right)=\int_{\Omega} p_{\omega}^{*}\left(\theta^{\prime}\right) p_{\theta}(\omega) d \mu(\omega) .
$$

Notice that $P^{*} P(\theta, \cdot)=P_{\theta}^{P^{*}}$. As a consequence of Fubini's Theorem, we get

$$
Q^{P^{*} P}=Q
$$

2) Because of (1), we introduce the notation $\Pi:=P \otimes Q$. So, (2) reads as $\Pi:=\beta_{Q}^{*} \otimes P^{*}$. Hence, after observing $\omega \in \Omega$, replacing the prior distribution $Q$ by the posterior distribution $P_{\omega}^{*}$, we get the probability distribution $\Pi_{\omega}:=$ $P \otimes P_{\omega}^{*}$ on $\mathcal{A} \otimes \mathcal{T}$. According to $(3), P P^{*}(\omega, A)=\Pi_{\omega}(A \times \Theta)=\Pi_{\omega}^{I}(A)$ where $I(\omega, \theta)=\omega$. This way the posterior predictive distribution $\left(P_{\omega}^{*}\right)^{P}$ given $\omega$ appears as the marginal $\Pi_{\omega}$-distribution on $\Omega$. 


\section{Bayesian estimation of probabilities, sampling distributions and densities}

According to Bayesian philosophy, given $A \in \mathcal{A}$, a natural estimator of $f_{A}(\theta):=P_{\theta}(A)$ is the posterior mean of $f_{A}$, which coincides with the posterior predictive probability of $A, T(\omega):=\left(P_{\omega}^{*}\right)^{P}(A)$. In fact, this is the Bayes estimator of $f_{A}$ (see Theorem 1.(i)).

So, the posterior predictive distribution $\left(P_{\omega}^{*}\right)^{P}$ appears as the natural Bayesian estimator of the probability distribution $P_{\theta}$.

To estimate probability measures, the squared total variation loss function

$$
W_{1}(Q, P):=\sup _{A \in \mathcal{A}}|Q(A)-P(A)|^{2},
$$

will be considered. An estimator of $f(\theta):=P_{\theta}$ is a Markov kernel $M$ : $(\Omega, \mathcal{A}) \longleftrightarrow(\Omega, \mathcal{A})$ so that, being observed $\omega \in \Omega, M(\omega, \cdot)$ is a probability measure on $\mathcal{A}$ which is considered as an estimation of $f$. We wonder if the Bayes mean risk of the estimator $M^{*}:=\left(P^{*}\right)^{P}$ is less than that of any other estimator $M$ of $f$, i.e., we wonder if

$$
\int_{\Omega \times \Theta} \sup _{A \in \mathcal{A}}\left|\left(P_{\omega}^{*}\right)^{P}(A)-P_{\theta}(A)\right|^{2} d \Pi(\omega, \theta) \leq \int_{\Omega \times \Theta} \sup _{A \in \mathcal{A}}\left|M(\omega, A)-P_{\theta}(A)\right|^{2} d \Pi(\omega, \theta) .
$$

Theorem 1.(ii) below gives the answer.

An estimator of the density $p_{\theta}$ on $\left(\Omega, \mathcal{A},\left\{P_{\theta}: \theta \in(\Theta, \mathcal{T}, Q)\right\}\right)$ is a measurable map $m:\left(\Omega^{2}, \mathcal{A}^{2}\right) \longrightarrow \mathbb{R}$ in such a way that, being observed $\omega \in \Omega$, the map $\omega^{\prime} \mapsto m\left(\omega, \omega^{\prime}\right)$ is an estimation of $p_{\theta}$.

It is well known (see Ghosal et al. (2017), p. 126) that, given two probability measures $Q$ and $P$ on $(\Omega, \mathcal{A})$ having densities $q$ and $p$ with respect to a $\sigma$-finite measure $\mu$,

$$
\sup _{A \in \mathcal{A}}|Q(A)-P(A)|=\frac{1}{2} \int|q-p| d \mu .
$$

So the Bayesian estimation of the sampling distribution $P_{\theta}$ for the squared total variation loss function corresponds to the Bayesian estimation of its density $p_{\theta}$ for the $L^{1}$-squared loss function

$$
W_{1}^{\prime}(q, p):=\left(\int|q-p| d \mu\right)^{2}
$$

The next Theorem also solves the estimation problem of the density. 
Theorem 1. Let $\left(\Omega, \mathcal{A},\left\{P_{\theta}: \theta \in(\Theta, \mathcal{T}, Q)\right\}\right)$ be a Bayesian statistical experiment dominated by a $\sigma$-finite measure $\mu$, where the $\sigma$-field $\mathcal{A}$ is supposed to be separable. We suppose that the likelihood function $\mathcal{L}(\omega, \theta):=p_{\theta}(\omega)=$ $d P_{\theta}(\omega) / d \mu$ is $\mathcal{A} \otimes \mathcal{T}$-measurable.

(i) Given $A \in \mathcal{A}$, the posterior predictive probability $\left(P_{\omega}^{*}\right)^{P}(A)$ of $A$ is the Bayes estimator of the probability $P_{\theta}(A)$ of $A$ for the squared error loss function

$$
W(x, \theta):=\left(x-P_{\theta}(A)\right)^{2} .
$$

Moreover, if $X$ is a real statistics with finite mean, its posterior predictive mean

$$
E_{\left(P_{\omega}^{*}\right)^{P}}(X)=\int_{\Theta} \int_{\Omega} X\left(\omega^{\prime}\right) d P_{\theta}\left(\omega^{\prime}\right) d P_{\omega}^{*}(\theta)
$$

is the Bayes estimator of $E_{\theta}(X)$.

(ii) The posterior predictive distribution $\left(P_{\omega}^{*}\right)^{P}$ is the Bayes estimator of the sampling distribution $P_{\theta}$ for the squared total variation loss function

$$
W_{1}(P, Q):=\sup _{A \in \mathcal{A}}|P(A)-Q(A)|^{2} .
$$

(iii) The posterior predictive density

$$
b_{Q, \omega}^{*}\left(\omega^{\prime}\right):=\frac{d\left(P_{\omega}^{*}\right)^{P}}{d \mu}\left(\omega^{\prime}\right)=\int_{\Theta} p_{\theta}\left(\omega^{\prime}\right) p_{\omega}^{*}(\theta) d Q(\theta) .
$$

is the Bayes estimator of the density $p_{\theta}$ for the $L^{1}$-squared loss function

$$
W_{1}^{\prime}(p, q):=\left(\int_{\Omega}|p-q| d \mu\right)^{2}
$$

\section{Bayesian estimation of sampling distributions and densities from a sample}

More generally, an estimator of $f(\theta):=P_{\theta}$ from a sample of size $n$ of this distribution is a Markov kernel

$$
M_{n}:\left(\Omega^{n}, \mathcal{A}^{n}\right) \succ(\Omega, \mathcal{A}) .
$$

Let us consider the Markov kernel

$$
P^{n}:(\Theta, \mathcal{T}) \succ\left(\Omega^{n}, \mathcal{A}^{n}\right)
$$


defined by $P^{n}(\theta, A)=P_{\theta}^{n}(A), A \in \mathcal{A}^{n}, \theta \in \Theta$. We write $\Pi_{n}:=P^{n} \otimes Q$, so that

$$
\Pi_{n}(A \times T)=\int_{T} P_{\theta}^{n}(A) d Q(\theta), \quad A \in \mathcal{A}^{n}, T \in \mathcal{T} .
$$

The corresponding prior predictive distribution is

$$
\beta_{Q, n}^{*}(A)=\int_{\Theta} P_{\theta}^{n}(A) d Q(\theta)=\Pi_{n}^{I}(A),
$$

where $I(\omega, \theta)=\omega$ for $\omega \in \Omega^{n}$. Let us write $I_{i}(\omega)=\omega_{i}$ and $\hat{I}_{i}(\omega, \theta)=\omega_{i}$, for $\omega \in \Omega^{n}$ and $i=1, \ldots, n$. Hence

$$
\left(\beta_{Q, n}^{*}\right)^{I_{i}}\left(A_{i}\right)=\int_{\Theta} P_{\theta}\left(A_{i}\right) d Q(\theta)=\beta_{Q}^{*}\left(A_{i}\right),
$$

and

$$
\Pi_{n}^{\hat{I}_{i}}\left(A_{i} \times T\right)=\int_{T} P_{\theta}\left(A_{i}\right) d Q(\theta)
$$

so

$$
\left(\beta_{Q, n}^{*}\right)^{I_{i}}=\beta_{Q}^{*}, \quad \text { and } \quad \Pi_{n}^{\hat{I}_{i}}=\Pi .
$$

Denoting $J(\omega, \theta)=\theta$, the posterior distribution $P_{\omega, n}^{*}:=\Pi_{n}^{J \mid I=\omega}, \omega \in \Omega^{n}$, is defined in such a way that

$$
\Pi_{n}(A \times T)=\int_{A} P_{\omega, n}^{*}(T) d \beta_{Q, n}^{*}(\omega) .
$$

The $\mu^{n}$-density of $P_{\theta}^{n}$ is

$$
p_{\theta, n}(\omega):=\frac{d P_{\theta}^{n}}{d \mu^{n}}(\omega)=\prod_{i=1}^{n} p_{\theta}\left(\omega_{i}\right) \quad \text { for } \omega=\left(\omega_{1}, \ldots, \omega_{n}\right) \in \Omega^{n} .
$$

The posterior density given $\omega \in \Omega^{n}$ is of the form

$$
p_{\omega, n}^{*}(\theta):=\frac{d P_{\omega, n}^{*}}{d Q}(\theta) \propto p_{\theta, n}(\omega) .
$$

According to Theorem 1.(ii), the Markov kernel

$$
\left(P_{n}^{*}\right)^{P^{n}}:\left(\Omega^{n}, \mathcal{A}^{n}\right) \succ\left(\Omega^{n}, \mathcal{A}^{n}\right)
$$

defined by

$$
\left(P_{n}^{*}\right)^{P^{n}}(\omega, A):=\left(P_{\omega, n}^{*}\right)^{P^{n}}(A)=\int_{\Theta} P_{\theta}^{n}(A) d P_{\omega, n}^{*}(\theta),
$$


is the Bayes estimator of the product probability measure $f_{n}(\theta):=P_{\theta}^{n}$. That is to say

$\int_{\Omega \times \Theta} \sup _{A \in \mathcal{A}^{n}}\left|\left(P_{\omega, n}^{*}\right)^{P^{n}}(A)-P_{\theta}^{n}(A)\right|^{2} d \Pi_{n}(\omega, \theta) \leq \int_{\Omega \times \Theta} \sup _{A \in \mathcal{A}^{n}}\left|M(\omega, A)-P_{\theta}^{n}(A)\right|^{2} d \Pi_{n}(\omega, \theta)$,

for every estimator $M:\left(\Omega^{n}, \mathcal{A}^{n}\right) \succ\left(\Omega^{n}, \mathcal{A}^{n}\right)$ of $P_{\theta}^{n}$.

The next theorem shows how marginalizing the posterior predictive distribution $\left(P_{\omega, n}^{*}\right)^{P^{n}}$ we can get the Bayes estimator of the sampling probability measure $P_{\theta}$ or its density.

Theorem 2. (Bayesian density estimation from a sample of size $n$ ) Let $\left(\Omega, \mathcal{A},\left\{P_{\theta}: \theta \in(\Theta, \mathcal{T}, Q)\right\}\right)$ be a Bayesian statistical experiment dominated by a $\sigma$-finite measure $\mu$, where the $\sigma$-field $\mathcal{A}$ is supposed to be separable. We suppose that the likelihood function $\mathcal{L}(\omega, \theta):=p_{\theta}(\omega)=d P_{\theta}(\omega) / d \mu$ is $\mathcal{A} \otimes \mathcal{T}$ measurable. Let $n \in \mathbb{N}$. All the estimation problems below are referred to the product Bayesian statistical experiment $\left(\Omega^{n}, \mathcal{A}^{n},\left\{P_{\theta}^{n}: \theta \in(\Theta, \mathcal{T}, Q)\right\}\right)$ corresponding to a $n$-sized sample of the observed unknown distribution. Let $I_{1}\left(\omega_{1}, \ldots, \omega_{n}\right):=\omega_{1}$.

(i) Given $A \in \mathcal{A}$,

$$
\left[\left(P_{\omega, n}^{*}\right)^{P^{n}}\right]^{I_{1}}(A)
$$

is the Bayes estimator of the probability $P_{\theta}(A)$ of $A$ for the squared error loss function

$$
W(x, \theta):=\left(x-P_{\theta}(A)\right)^{2} .
$$

(ii) The distribution

$$
\left[\left(P_{\omega, n}^{*}\right)^{P^{n}}\right]^{I_{1}}
$$

of the projection $I_{1}$ under the posterior predictive probability $\left(P_{\omega, n}^{*}\right)^{P^{n}}$ is the Bayes estimator of the sampling distribution $P_{\theta}$ for the squared total variation loss function

$$
W_{1}(P, Q):=\sup _{A \in \mathcal{A}}|P(A)-Q(A)|^{2} .
$$

(iii) The marginal posterior predictive density

$$
b_{Q, \omega, n}^{*}\left(\omega^{\prime}\right):=\frac{d\left[\left(P_{\omega, n}^{*}\right)^{P^{n}}\right]^{I_{1}}}{d \mu}\left(\omega^{\prime}\right)=\int_{\Theta} p_{\theta}\left(\omega^{\prime}\right) p_{\omega, n}^{*}(\theta) d Q(\theta) .
$$

is the Bayes estimator of the density $p_{\theta}$ for the $L^{1}$-squared loss function

$$
W_{1}^{\prime}(p, q):=\left(\int_{\Omega}|p-q| d \mu\right)^{2}
$$


We end this section with a remark that address the problem of estimating a real distribution function.

Remark. (Bayesian estimation of a distribution function) When $P_{\theta}$ is a probability distribution on the line, we may be interested in the estimation of its distribution function $\left.\left.F_{\theta}(t):=P_{\theta}(]-\infty, t\right]\right)$. An estimator of such a distribution function is a map

$$
\left.\left.F:(x, t) \in \mathbb{R}^{n} \times \mathbb{R} \longmapsto F(x, t):=M(x,]-\infty, t\right]\right)
$$

for a Markov kernel $M:\left(\mathbb{R}^{n}, \mathcal{R}^{n}\right) \succ(\mathbb{R}, \mathcal{R})$, where $\mathcal{R}$ denotes the Borel $\sigma$-field on $\mathbb{R}$.

Accordig to the previous results, given $t \in \mathbb{R}$,

$$
\left.\left.F_{x}^{*}(t):=\left[\left(P_{x, n}^{*}\right)^{P^{n}}\right]^{I_{1}}(]-\infty, t\right]\right)=\int_{-\infty}^{t} \int_{\Theta} p_{\theta, n}(y) \cdot p_{x, n}^{*}(\theta) d Q(\theta) d \mu^{n}(y)
$$

is the Bayes estimator of $F_{\theta}(t)$ for the squared error loss function. So

$$
\int_{\mathbb{R}^{n} \times \Theta}\left|F_{x}^{*}(t)-F_{\theta}(t)\right|^{2} d \Pi(x, \theta) \leq \int_{\mathbb{R}^{n} \times \Theta}\left|F(x, t)-F_{\theta}(t)\right|^{2} d \Pi(x, \theta)
$$

for any other estimator $F$ of $F_{\theta}$. Since

$$
\sup _{t \in \mathbb{R}}\left|F(x, t)-F_{\theta}(t)\right|=\sup _{r \in \mathbb{Q}}\left|F(x, r)-F_{\theta}(r)\right|
$$

we have that, given $(x, \theta) \in \mathbb{R}^{n} \times \Theta$ and $k \in \mathbb{N}$, there exists $r_{k} \in \mathbb{Q}$ such that

$$
C(x, \theta)-\frac{1}{k} \leq\left|F_{x}^{*}\left(r_{k}\right)-F_{\theta}\left(r_{k}\right)\right|,
$$

where $C(x, \theta):=\sup _{t \in \mathbb{R}}\left|F_{x}^{*}(t)-F_{\theta}(t)\right|^{2}$, and hence (see the remark at the end of Section 5)

$$
\begin{aligned}
\int_{\mathbb{R}^{n} \times \Theta} C(x, \theta) d \Pi(x, \theta) & \leq \int_{\mathbb{R}^{n} \times \Theta}\left|F_{x}^{*}\left(r_{k}\right)-F_{\theta}\left(r_{k}\right)\right|^{2} d \Pi(x, \theta)+\frac{1}{k} \\
& \leq \int_{\mathbb{R}^{n} \times \Theta} \sup _{t \in \mathbb{R}}\left|F(x, t)-F_{\theta}(t)\right|^{2} d \Pi(x, \theta)+\frac{1}{k} .
\end{aligned}
$$

We have proved that the posterior predictive distribution function $F_{x}^{*}$ is the Bayes estimator of the distribution function $F_{\theta}$ for the $L^{\infty}$-squared loss function

$$
W^{\prime \prime}(F, G)=\left(\sup _{t \in \mathbb{R}}|F(t)-G(t)|\right)^{2}
$$




\section{Consistency of the Bayesian estimator of the density}

First we adapt the framework to an asymptotic context. Let

$$
\left(\Omega, \mathcal{A},\left\{P_{\theta}: \theta \in(\Theta, \mathcal{T}, Q)\right\}\right)
$$

be a Bayesian experiment and consider the product Bayesian experiment

$$
\left(\Omega^{\mathbb{N}}, \mathcal{A}^{\mathbb{N}},\left\{P_{\theta}^{\mathbb{N}}: \theta \in(\Theta, \mathcal{T}, Q)\right\}\right)
$$

corresponding to an infinite sample of the unknown distribution $P_{\theta}$. Let us write

$I(\omega, \theta):=\omega, \quad J(\omega, \theta):=\theta, \quad I_{n}(\omega, \theta):=\omega_{n} \quad$ and $\quad I_{(n)}(\omega):=\omega_{(n)}:=\left(\omega_{1}, \ldots, \omega_{n}\right)$,

where $n \in \mathbb{N}$.

We suppose that $P^{\mathbb{N}}(\theta, A):=P_{\theta}^{\mathbb{N}}(A)$ is a Markov kernel. Let

$$
\Pi_{\mathbb{N}}:=P^{\mathbb{N}} \otimes Q
$$

i.e.,

$$
\Pi_{\mathbb{N}}(A \times T)=\int_{T} P_{\theta}^{\mathbb{N}}(A) d Q(\theta), \quad A \in \mathcal{A}, T \in \mathcal{T} .
$$

Being $Q:=\Pi_{\mathbb{N}}^{J}, P_{\theta}^{\mathbb{N}}$ is a version of the conditional distribution (regular conditional probability) $\Pi_{\mathbb{N}}^{I \mid J=\theta}$. Analogously, $P_{\theta}^{n}$ is a version of the conditional distribution $\Pi_{\mathbb{N}}^{I_{(n)} \mid J=\theta}$.

Let $\beta_{Q, \mathbb{N}}^{*}:=\Pi_{\mathbb{N}}^{I}$, the prior predictive distribution in $\Omega^{\mathbb{N}}$. Similarly we write $\beta_{Q, n}^{*}:=\Pi_{\mathbb{N}}^{I_{(n)}}$ for the prior predictive distribution in $\Omega^{n}$. So, the posterior distribution is $P_{\omega, \mathbb{N}}^{*}:=\Pi_{\mathbb{N}}^{J \mid I=\omega}$, in such a way that

$$
\Pi_{\mathbb{N}}(A \times T)=\int_{T} P_{\theta}^{\mathbb{N}}(A) d Q(\theta)=\int_{A} P_{\omega, \mathbb{N}}^{*}(T) d \beta_{Q, \mathbb{N}}^{*}(\omega), \quad A \in \mathcal{A}, T \in \mathcal{T} .
$$

Denote $P_{\omega_{(n)}, n}^{*}:=\Pi_{\mathbb{N}}^{J \mid I_{(n)}=\omega_{(n)}}$ for $\omega_{(n)} \in \Omega^{n}$.

Write $P_{\omega_{(n)}, n}^{* P}$ for the posterior predictive distribution given $\omega_{(n)} \in \Omega^{n}$, defined for $A \in \mathcal{A}$ as

$$
P_{\omega_{(n)}, n}^{* P}(A)=\int_{\Theta} P_{\theta}(A) d P_{\omega_{(n)}, n}^{*}(\theta) .
$$


In the dominated case we can assume without loss of generality that the dominating measure $\mu$ is a probability measure (because of (1) below). We write $p_{\theta}=d P_{\theta} / d \mu$.

We have that, far all $n$ and every event $A \in \mathcal{A}$,

$$
\begin{aligned}
P_{\omega_{(n)}, n}^{* P}(A) & =\int_{\Theta} P_{\theta}(A) d P_{\omega_{(n)}, n}^{*}(\theta)=\int_{\Theta} \int_{A} p_{\theta}\left(\omega^{\prime}\right) d \mu\left(\omega^{\prime}\right) d P_{\omega_{(n)}, n}^{*}(\theta) \\
& =\int_{A} \int_{\Theta} p_{\theta}\left(\omega^{\prime}\right) d P_{\omega_{(n)}, n}^{*}(\theta) d \mu\left(\omega^{\prime}\right)
\end{aligned}
$$

which proves that

$$
p_{\omega_{(n)}, n}^{* P}\left(\omega^{\prime}\right):=\int_{\Theta} p_{\theta}\left(\omega^{\prime}\right) d P_{\omega_{(n)}, n}^{*}(\theta)
$$

is a $\mu$-density of $P_{\omega_{(n)}, n}^{*}$.

In the same way

$$
p_{\omega, \mathbb{N}}^{*}\left(\omega^{\prime}\right):=\int_{\Theta} p_{\theta}\left(\omega^{\prime}\right) d P_{\omega, \mathbb{N}}^{*}(\theta)
$$

is a $\mu$-density of $P_{\omega, \mathbb{N}}^{*}$, the posterior predictive distribution given $\omega \in \Omega^{\mathbb{N}}$.

It has been previously proved by the author (see Nogales (2020)) that the posterior predictive distribution $P_{\omega_{(n)}, n}^{* P}$ (resp. the posterior predictive density $p_{\omega_{(n)}, n}^{*}$ ) is the Bayes estimator of the sampling distribution $P_{\theta}$ (resp. the density $p_{\theta}$ ) for the squared variation total (resp. the squared $\mathbb{L}^{1}$ ) loss function in the product experiment $\left(\Omega^{n}, \mathcal{A}^{n},\left\{P_{\theta}^{n}: \theta \in(\Theta, \mathcal{T}, Q)\right\}\right)$. Analogously, the posterior predictive distribution $P_{\omega, \mathbb{N}}^{*}$ (resp. the posterior predictive density $\left.p_{\omega, \mathbb{N}}^{*}\right)$ is the Bayes estimator of the sampling distribution $P_{\theta}$ (resp. the density $p_{\theta}$ ) for the squared variation total (resp. the squared $\mathbb{L}^{1}$ ) loss function in the product experiment $\left(\Omega^{\mathbb{N}}, \mathcal{A}^{\mathbb{N}},\left\{P_{\theta}^{\mathbb{N}}: \theta \in(\Theta, \mathcal{T}, Q)\right\}\right)$.

It is well known that

$$
\sup _{A \in \mathcal{A}}\left|P_{\omega_{(n)}, n}^{* P}(A)-P_{\theta}(A)\right|=\frac{1}{2} \int_{\Omega}\left|p_{\omega_{(n)}, n}^{* P}-p_{\theta}\right| d \mu .
$$

We wonder if the Bayes risk of the Bayes estimator $P_{\omega_{(n)}, n}^{*}$ of the sampling distribution $P_{\theta}$ goes to zero when $n \rightarrow \infty$, i.e., if

$$
\lim _{n} \int_{\Omega^{\mathbb{N}} \times \Theta} \sup _{A \in \mathcal{A}}\left|P_{\omega_{(n)}, n}^{*} P^{P}(A)-P_{\theta}(A)\right| d \Pi_{\mathbb{N}}(\omega, \theta)=0 .
$$


In terms of densities, the question is whether the Bayes risk of the Bayes estimator $p_{\omega_{(n)}, n}^{* P}$ of the density $p_{\theta}$ goes to zero when $n \rightarrow \infty$, i.e., if

$$
\lim _{n} \int_{\Omega^{\mathbb{N}} \times \Theta}\left(\int_{\Omega}\left|p_{\omega_{(n)}, n}^{*}\left(\omega^{\prime}\right)-p_{\theta}\left(\omega^{\prime}\right)\right| d \mu\left(\omega^{\prime}\right)\right)^{2} d \Pi_{\mathbb{N}}(\omega, \theta)=0 .
$$

The following result, consequence of a Theorem of Doob that the reader can find in Ghosal et al. (2017), provides the answers and shows the consistency of the estimator.

Theorem 3. Let $\left(\Omega, \mathcal{A},\left\{P_{\theta}: \theta \in(\Theta, \mathcal{T}, Q)\right\}\right)$ be a Bayesian experiment dominated by a $\sigma$-finite measure $\mu$. Let us suppose that $(\Omega, \mathcal{A})$ is a Borel standar space, that $\Theta$ is a Borel subset of a polish space and $\mathcal{T}$ is its Borel $\sigma$-field. Suppose also that the likelihood function $\mathcal{L}(\omega, \theta):=p_{\theta}(\omega)=\frac{d P_{\theta}}{d \mu}(\omega)$ is $\mathcal{A} \times \mathcal{T}$-measurable and the family $\left\{P_{\theta}: \theta \in \Theta\right\}$ is identifiable. Then:

(a) The posterior predictive density $p_{\omega_{(n)}, n}^{P}$ is the Bayes estimator of the density $p_{\theta}$ in the product experiment $\left(\Omega^{n}, \mathcal{A}^{n},\left\{P_{\theta}^{n}: \theta \in(\Theta, \mathcal{T}, Q)\right\}\right)$ for the squared $\mathbb{L}^{1}$ loss function. Moreover the risk function converges to 0 both for the $\mathbb{L}^{1}$ loss function and the squared $\mathbb{L}^{1}$ loss function.

(b) The posterior predictive distribution $P_{\omega_{(n)}, n}^{P}$ is the Bayes estimator of the sampling distribution $P_{\theta}$ in the product experiment $\left(\Omega^{n}, \mathcal{A}^{n},\left\{P_{\theta}^{n}: \theta \in\right.\right.$ $(\Theta, \mathcal{T}, Q)\})$ for the squared variation total loss function. Moreover the risk function converges to 0 both for the variation total loss function and the squared variation total loss function.

(c) The posterior predictive density is a consistent estimator of the density $p_{\theta}$, i.e.,

$$
\lim _{n} p_{\omega_{(n)}, n}^{*}\left(\omega^{\prime}\right)=p_{\theta}\left(\omega^{\prime}\right), \quad \mu \times P_{\theta}^{\mathbb{N}}-\text { a.e. }
$$

for $Q$-almost every $\theta \in \Theta$.

\section{Examples}

Example 1. Let $G(\alpha, \beta)$ be the distribution gamma with parameters $\alpha, \beta>$ 0 and $P_{\theta}:=G\left(1, \theta^{-1}\right)$, whose density is $p_{\theta}(x)=\theta \exp \{-\theta x\}$ for $x>0$.

So $P_{\theta}^{n}$ is the joint distribution of a sample of size $n$ of an exponential distribution of parameter $1 / \theta$ and its density is $p_{\theta, n}(x)=\theta^{n} \exp \left\{-\theta \sum_{i} x_{i}\right\}$ for $x=\left(x_{1}, \ldots, x_{n}\right) \in \mathbb{R}_{+}^{n}$.

Consider the prior distribution $Q:=G\left(1, \lambda^{-1}\right)$ for some known $\lambda>0$. 
Since, for $a>0$,

$$
\int_{0}^{\infty} \theta^{n} \exp \{-a \theta\} d \theta=\frac{n !}{a^{n+1}}
$$

we have that the posterior density given $x \in \mathbb{R}_{+}^{n}$ is

$$
p_{x, n}^{*}(\theta)=\frac{\lambda}{n !}\left(\sum_{i} x_{i}\right)^{n+1} \theta^{n} \exp \left\{-\theta\left(\lambda+\sum_{i} x_{i}\right)\right\} .
$$

So, denoting by $\mu_{n}$ the Lebesgue measure on $\mathbb{R}_{+}^{n}$, the density of the posterior predictive probability given $x$ is

$$
\frac{d\left(P_{x, n}^{*}\right)^{P^{n}}}{d \mu_{n}}\left(x^{\prime}\right)=\int_{\Theta} p_{\theta, n}\left(x^{\prime}\right) \cdot p_{x, n}^{*}(\theta) d \theta=\lambda \frac{(2 n) !}{n !} \frac{\left(\sum_{i} x_{i}\right)^{n+1}}{\left(\lambda+\sum_{i} x_{i}^{\prime}+\sum_{i} x_{i}\right)^{2 n+1}} .
$$

According to the previous results, this is the Bayes estimator of the joint density $p_{\theta, n}$ for the loss function

$$
W_{n}^{\prime}(q, p):=\left(\int_{\mathbb{R}^{n}}|q-p| d \mu_{n}\right)^{2}
$$

while the posterior predictive distribution $\left(P_{x, n}^{*}\right)^{P^{n}}$ is the Bayes estimator of the sampling distribution $P_{\theta}^{n}$ for the squared total variation loss function on $\left(\Omega^{n}, \mathcal{A}^{n}\right)$.

Moreover, the image $M_{n}^{*}(x, \cdot):=\left[\left(P_{x, n}^{*}\right)^{P^{n}}\right]^{I_{1}}=I_{1}\left(P_{x, n}^{*}\right)^{P^{n}}$ is the Bayes estimator of the probability distribution $P_{\theta}$ for the squared total variation on $(\Omega, \mathcal{A})$ and its density

$x^{\prime}>0 \longmapsto \frac{d M_{n}^{*}(x, \cdot)}{d \mu_{1}}\left(x^{\prime}\right)=\int_{0}^{\infty} p_{\theta}\left(x^{\prime}\right) \cdot p_{x, n}^{*}(\theta) d \theta=\frac{n \lambda\left(\sum_{i=1}^{n} x_{i}\right)^{n+1}}{\left(\lambda+x^{\prime}+\sum_{i=1}^{n} x_{i}\right)^{n+2}}$

is the Bayes estimator of the density $p_{\theta}$ for the $L^{1}$-squared loss function $W_{1}^{\prime}$.

Example 2. Let $P_{\theta}$ the normal distribution $N\left(\theta, \sigma_{0}^{2}\right)$ with unknown mean $\theta \in \mathbb{R}$ and known variance $\sigma_{0}^{2}$. Let $Q:=N\left(\mu, \tau^{2}\right)$ be the prior distribution where the mean $\mu$ and variance $\tau^{2}$ are known constants. It is well known that the posterior distribution is $P_{x, n}^{*}=N\left(m_{n}(x), s_{n}^{2}\right)$ where

$$
m_{n}(x)=\frac{n \tau^{2} \bar{x}+\sigma_{0}^{2} \mu}{n \tau^{2}+\sigma_{0}^{2}} \quad \text { and } \quad s_{n}^{2}=\frac{\tau^{2} \sigma_{0}^{2}}{n \tau^{2}+\sigma_{0}^{2}} .
$$


It can be shown that the distributiion of $I_{1}$ with respect to the posterior predictive distribution is

$$
\left[\left(P_{x, n}^{*}\right)^{P^{n}}\right]=N\left(m_{n}(x), \sigma_{0}^{2}+s_{n}^{2}\right) .
$$

For the details, the reader is addressed to Boldstat (2004, p. 185), where the distributiion of $I_{1}$ with respect to the posterior predictive distribution is referred to as the predcitive distribution for the next observation given the observation $x$.

So $M_{n}^{*}(x, \cdot):=N\left(m_{n}(x), \sigma_{0}^{2}+s_{n}^{2}\right)$ is the Bayes estimator of the sampling distribution $N\left(\theta, \sigma_{0}^{2}\right)$ for the squared total variation loss function and the density of $N\left(m_{n}(x), \sigma_{0}^{2}+s_{n}^{2}\right)$ is the Bayes estimator of the density of $N\left(\theta, \sigma_{0}^{2}\right)$ for the $L^{1}$-squared loss function.

Example 3. Let $P_{\theta}$ be the Poisson distribution with parameter $\theta>0$ whose probablity function (or density with respect to the counter measure $\mu_{1}$ on $\left.\mathbb{N}_{0}\right)$ is $p_{\theta}(k)=\exp \{-\theta\} \frac{\theta^{k}}{k !}$ for $k \in \mathbb{N}_{0}$.

So $P_{\theta}^{n}$ is the joint distribution of a sample of size $n$ of a Poisson distribution of parameter $\theta$ and its probability function (or density with respecto to the counter measure $\mu_{n}$ on $\left.\mathbb{N}_{0}^{n}\right)$ is $p_{\theta, n}(k)=\exp \{-n \theta\} \frac{\theta^{\|k\|_{1}}}{\prod_{i=1}^{n}\left(k_{i} !\right)}$ for $k=\left(k_{1}, \ldots, k_{n}\right) \in \mathbb{N}_{0}^{n}$, where $\|k\|_{1}:=\sum_{i=1}^{n} k_{i}$.

Consider the prior distribution $Q:=G\left(1, \lambda^{-1}\right)$ for some known $\lambda>0$.

It is readily shown that the posterior distribution given $k \in \mathbb{N}_{0}^{n}$ is the gamma distribution $G\left(\|k\|_{1}, \frac{1}{\lambda+n}\right)$ whose density is

$$
p_{k, n}^{*}(\theta)=\frac{(\lambda+n)^{\|k\|_{1}}}{\left(\|k\|_{1}\right) !} \cdot \theta^{\|k\|_{1}} \exp \{-\theta(\lambda+n)\} .
$$

So the probability function of the posterior predictive probability given $k \in$ $\mathbb{N}_{0}^{n}$ is

$\frac{d\left(P_{k, n}^{*}\right)^{P^{n}}}{d \mu_{n}}\left(k^{\prime}\right)=\int_{\Theta} p_{\theta, n}\left(k^{\prime}\right) \cdot p_{k, n}^{*}(\theta) d \theta=\frac{\left(\left\|k^{\prime}\right\|_{1}+\|k\|_{1}\right) !}{\prod_{i=1}^{n}\left(k_{i} !\right) \cdot\left(\|k\|_{1}\right) !} \cdot \frac{(\lambda+n)^{\|k\|_{1}+1}}{(\lambda+2 n)^{\left\|k^{\prime}\right\|_{1}+\|k\|_{1}+1}}$.

According to the previous results, this is the Bayes estimator of the joint density $p_{\theta, n}$ for the loss function

$$
W_{n}^{\prime}(q, p):=\left(\int_{\mathbb{N}_{0}^{n}}|q-p| d \mu_{n}\right)^{2}
$$


while the posterior predictive distribution $\left(P_{k, n}^{*}\right)^{P^{n}}$ is the Bayes estimator of the sampling distribution $P_{\theta}^{n}$ for the squared total variation loss function on $\mathbb{N}_{0}^{n}$.

Moreover, the image $M_{n}^{*}(k, \cdot):=\left[\left(P_{k, n}^{*}\right)^{P^{n}}\right]^{I_{1}}=I_{1}\left(P_{k, n}^{*}\right)^{P^{n}}$ is the Bayes estimator of the probability distribution $P_{\theta}$ for the squared total variation on $\mathbb{N}_{0}$ and its probability function

$k^{\prime} \geq 0 \longmapsto \frac{d M_{n}^{*}(k, \cdot)}{d \mu_{1}}\left(k^{\prime}\right)=\int_{0}^{\infty} p_{\theta}\left(k^{\prime}\right) \cdot p_{k, n}^{*}(\theta) d \theta=\frac{\left(k^{\prime}+\|k\|_{1}\right) !}{k^{\prime} ! \cdot\left(\|k\|_{1}\right) !} \cdot \frac{(\lambda+n)^{\|k\|_{1}+1}}{(\lambda+n+1)^{k^{\prime}+\|k\|_{1}+1}}$

is the Bayes estimator of the probability function $p_{\theta}$ for the loss function $W_{1}^{\prime}$.

Example 4. Let $P_{\theta}$ be the Bernoulli distribution with parameter $\theta \in(0,1)$ whose probability function is $p_{\theta}(k):=\theta^{k}(-\theta)^{n-k}, k=0,1$. So $P_{\theta}^{n}$ is the joint distribution of a sample of size $n$ of a Bernoulli distribution with parameter $\theta$ and its probability function is

$$
p_{\theta, n}(k)=\theta^{\|k\|_{1}}(1-\theta)^{n-\|k\|_{1}}, \quad k \in\{0,1\}^{n}
$$

where $\|k\|_{1}:=\sum_{i=1}^{k} k_{i}$. Consider the uniform distribution on the unit interval as prior distribution. So, the posterior distribution given $k \in\{0,1\}^{n}$ is the Beta distribution

$$
P_{k, n}^{*}=B\left(\|k\|_{1}+1, n-\|k\|_{1}+1\right)
$$

with parameters $\|k\|_{1}+1$ and $n-\|k\|_{1}+1$. Hence, denoting $\mu_{n}$ for the counter measure on $\{0,1\}^{n}$ and $\beta$ the Euler beta function, the probability function of the posterior predictive probability given $k \in\{0,1\}^{n}$ is

$$
\begin{aligned}
\frac{d\left(P_{k, n}^{*}\right)^{P^{n}}}{d \mu_{n}}\left(k^{\prime}\right) & =\int_{\Theta} p_{\theta, n}\left(k^{\prime}\right) \cdot p_{k, n}^{*}(\theta) d \theta \\
& =\frac{\beta\left(\|k\|_{1}+\left\|k^{\prime}\right\|_{1}+1\right) \beta\left(2 n-\|k\|_{1}-\left\|k^{\prime}\right\|_{1}+1\right)}{\beta\left(\|k\|_{1}+1\right) \beta\left(n-\|k\|_{1}+1\right)} \\
& =\frac{\Gamma(n+2)}{\Gamma(2 n+2)} \cdot \frac{\left(\left\|k^{\prime}\right\|_{1}+\|k\|_{1}\right) ! \cdot\left(2 n-\left\|k^{\prime}\right\|_{1}-\|k\|_{1}\right) !}{\left(\|k\|_{1}\right) ! \cdot\left(n-\|k\|_{1}\right) !} .
\end{aligned}
$$

This is the Bayes estimator of the joint probability function $p_{\theta, n}$ for the loss function $W_{n}^{\prime}(q, p):=\left(\int_{\{0,1\}^{n}}|q-p| d \mu_{n}\right)^{2}$, while the posterior predictive distribution $\left(P_{k, n}^{*}\right)^{P^{n}}$ is the Bayes estimator of the sampling distribution $P_{\theta}^{n}$ for the squared total variation loss function on $\{0,1\}^{n}$. 
Moreover, the image $M_{n}^{*}(k, \cdot):=\left[\left(P_{k, n}^{*}\right)^{P^{n}}\right]^{I_{1}}=I_{1}\left(P_{k, n}^{*}\right)^{P^{n}}$ is the Bayes estimator of the probability distribution $P_{\theta}$ for the squared total variation on $\{0,1\}$ and its probability function

$$
\begin{aligned}
k^{\prime} \in\{0,1\} \longmapsto & \frac{d M_{n}^{*}(k, \cdot)}{d \mu_{1}}\left(k^{\prime}\right)=\int_{0}^{\infty} p_{\theta}\left(k^{\prime}\right) \cdot p_{k, n}^{*}(\theta) d \theta \\
& =\frac{\Gamma(n+2)}{\Gamma(2 n+2)} \cdot \frac{\left(k^{\prime}+\|k\|_{1}\right) ! \cdot\left(2 n-k^{\prime}-\|k\|_{1}\right) !}{\left(\|k\|_{1}\right) ! \cdot\left(n-\|k\|_{1}\right) !}
\end{aligned}
$$

is the Bayes estimator of the probability function $p_{\theta}$ for the $L^{1}$-squared loss function $W_{1}^{\prime}$.

\section{Proofs}

Proof. (of Theorem 1) (i) Notice that, writing $f_{A}(\theta):=P_{\theta}(A)$,

$$
\left(P_{\omega}^{*}\right)^{P}(A)=\int_{\Theta} P_{\theta}(A) d P_{\omega}^{*}(\theta)=E_{P_{\omega}^{*}}\left(f_{A}\right)
$$

that, as it is well known (see Nogales (1998) p. 147), is the Bayes estimator of $f_{A}$ for the squared error loss function.

In the same way, if $X$ is a real integrable statistic on $(\Omega, \mathcal{A})$ and $f(\theta):=$ $E_{\theta}(X)$, we have that

$$
E_{\left(P_{\omega}^{*}\right)^{P}}(X)=\int_{\Theta} \int_{\Omega} X\left(\omega^{\prime}\right) d P_{\theta}\left(\omega^{\prime}\right) d P_{\omega}^{*}(\theta)=E_{P_{\omega}^{*}}(f)
$$

is the Bayes estimator of $f$, the mean of $X$.

(ii) According (i), given $A \in \mathcal{A}$,

$$
\int_{\Omega \times \Theta}\left|\left(P_{\omega}^{*}\right)^{P}(A)-P_{\theta}(A)\right|^{2} d \Pi(\omega, \theta) \leq \int_{\Omega \times \Theta}\left|X(\omega)-P_{\theta}(A)\right|^{2} d \Pi(\omega, \theta),
$$

for any real measurable function $X$ on $(\Omega, \mathcal{A})$. If $\mathcal{A}$ is a separable $\sigma$-field, there exists a countable algebra $\mathcal{A}_{0}$ such that $\mathcal{A}=\sigma\left(\mathcal{A}_{0}\right)$. In particular, it follows that

$$
\sup _{A \in \mathcal{A}}\left|M(\omega, A)-P_{\theta}(A)\right|^{2}=\sup _{A \in \mathcal{A}_{0}}\left|M(\omega, A)-P_{\theta}(A)\right|^{2}
$$

is $(\mathcal{A} \otimes \mathcal{T})$-measurable. Given $(\omega, \theta) \in \Omega \times \Theta$, let

$$
C(\omega, \theta):=\sup _{A \in \mathcal{A}}\left|\left(P_{\omega}^{*}\right)^{P}(A)-P_{\theta}(A)\right|^{2}
$$


and, given $n \in \mathbb{N}$, choose $A_{n} \in \mathcal{A}_{0}$ so that

$$
C-\frac{1}{n} \leq\left|\left(P_{\omega}^{*}\right)^{P}\left(A_{n}\right)-P_{\theta}\left(A_{n}\right)\right|^{2} .
$$

It follows from this that

$$
\begin{aligned}
\int_{\Omega \times \Theta} C d \Pi & \leq \int_{\Omega \times \Theta}\left|\left(P_{\omega}^{*}\right)^{P}\left(A_{n}\right)-P_{\theta}\left(A_{n}\right)\right|^{2} d \Pi(\omega, \theta)+\frac{1}{n} \\
& \leq \int_{\Omega \times \Theta} \sup _{A \in \mathcal{A}}\left|M(\omega, A)-P_{\theta}(A)\right|^{2} d \Pi(\omega, \theta)+\frac{1}{n}
\end{aligned}
$$

and this gives the proof as $n$ is arbitrary. To refine the proof from a measuretheoretical point of view, a judicious use of the Ryll-Nardzewski and Kuratowski measurable selection theorem would also be helpful. See the details in the remark at the end of the section.

(iii) It follows from (ii) that, to estimate the density $p_{\theta}$, the posterior predictive density

$$
b_{Q, \omega}^{*}\left(\omega^{\prime}\right):=\frac{d\left(P_{\omega}^{*}\right)^{P}}{d \mu}\left(\omega^{\prime}\right)
$$

minimizes the Bayes mean risk for the loss function

$$
W_{1}^{\prime}(q, p):=\left(\int|q-p| d \mu\right)^{2}
$$

i.e.,

$$
E_{\Pi}\left[\left(\int\left|b_{Q, \omega}^{*}-p_{\theta}\right| d \mu\right)^{2}\right] \leq E_{\Pi}\left[\left(\int\left|m(\omega, \cdot)-p_{\theta}\right| d \mu\right)^{2}\right]
$$

for any measurable function $m: \Omega \times \Omega \rightarrow[0, \infty)$ such that $\int_{\Omega} m\left(\omega, \omega^{\prime}\right) d \mu\left(\omega^{\prime}\right)=$ 1 for every $\omega$.

Proof. (of Theorem 2) (i) Given $A \in \mathcal{A}^{n}$, Theorem 1.(i) shows that the posterior predictive probability $\left(P_{\omega, n}^{*}\right)^{P^{n}}(A)$ of $A$ is the Bayes estimator of $f_{A}(\theta):=P_{\theta}^{n}(A)$ in the product Bayesian statistical experiment, as

$$
\left(P_{\omega, n}^{*}\right)^{P^{n}}(A)=\int_{\Theta} P_{\theta}^{n}(A) d P_{\omega, n}^{*}(\theta)=E_{P_{\omega, n}^{*}}\left(f_{A}\right),
$$

i.e.

$\int_{\Omega^{n} \times \Theta}\left|\left(P_{\omega, n}^{*}\right)^{P^{n}}(A)-P_{\theta}^{n}(A)\right|^{2} d \Pi_{n}(\omega, \theta) \leq \int_{\Omega^{n} \times \Theta}\left|X(\omega)-P_{\theta}^{n}(A)\right|^{2} d \Pi_{n}(\omega, \theta)$ 
for any other estimator $X:\left(\Omega^{n}, \mathcal{A}^{n}\right) \rightarrow \mathbb{R}$ of $f_{A}$. In particular, given $A \in \mathcal{A}$, applying this result to $I_{1}^{-1}(A)=A \times \Omega^{n-1} \in \mathcal{A}^{n}$, we obtain that

$$
\int_{\Omega^{n} \times \Theta}\left|\left(P_{\omega, n}^{*}\right)^{P^{n}}\left(I_{1}^{-1}(A)\right)-P_{\theta}(A)\right|^{2} d \Pi_{n}(\omega, \theta) \leq \int_{\Omega^{n} \times \Theta}\left|X(\omega)-P_{\theta}(A)\right|^{2} d \Pi_{n}(\omega, \theta)
$$

for any other estimator $X:\left(\Omega^{n}, \mathcal{A}^{n}\right) \rightarrow \mathbb{R}$ of $g_{A}:=P_{\theta}(A)$.

(ii) Being $\mathcal{A}$ a separable $\sigma$-field, there exists a countable algebra $\mathcal{A}_{0}$ such that $\mathcal{A}=\sigma\left(\mathcal{A}_{0}\right)$. In particular, it follows that

$$
\sup _{A \in \mathcal{A}}\left|M(\omega, A)-P_{\theta}(A)\right|^{2}=\sup _{A \in \mathcal{A}_{0}}\left|M(\omega, A)-P_{\theta}(A)\right|^{2}
$$

is $(\mathcal{A} \otimes \mathcal{T})$-measurable. Given $(\omega, \theta) \in \Omega^{n} \times \Theta$, let

$$
C_{n}(\omega, \theta):=\sup _{A \in \mathcal{A}}\left|\left(P_{\omega, n}^{*}\right)^{P^{n}}\left(I_{1}^{-1}(A)\right)-P_{\theta}(A)\right|^{2}
$$

and, given $k \in \mathbb{N}$, choose $A_{k} \in \mathcal{A}_{0}$ so that

$$
C_{n}-\frac{1}{k} \leq\left|\left(P_{\omega, n}^{*}\right)^{P^{n}}\left(I_{1}^{-1}\left(A_{k}\right)\right)-P_{\theta}\left(A_{k}\right)\right|^{2} .
$$

It follows that

$$
\begin{aligned}
\int_{\Omega \times \Theta} C_{n} d \Pi_{n} & \leq \int_{\Omega \times \Theta}\left|\left(P_{\omega, n}^{*}\right)^{P^{n}}\left(I_{1}^{-1}\left(A_{k}\right)\right)-P_{\theta}\left(A_{k}\right)\right|^{2} d \Pi_{n}(\omega, \theta)+\frac{1}{k} \\
& \leq \int_{\Omega \times \Theta} \sup _{A \in \mathcal{A}}\left|M(\omega, A)-P_{\theta}(A)\right|^{2} d \Pi_{n}(\omega, \theta)+\frac{1}{k}
\end{aligned}
$$

for any Markov kernel $M:\left(\Omega^{n}, \mathcal{A}^{n}\right) \succ(\Omega, \mathcal{A})$ and, being $k$ arbitrary, this proves that

$$
M_{n}^{*}(\omega, A):=\left(P_{\omega, n}^{*}\right)^{P^{n}}\left(I_{1}^{-1}(A)\right)
$$

is the Bayes estimator of $f(\theta):=P_{\theta}$ for the squared total variation loss function in the Bayesian statistical experiment

$$
\left(\Omega^{n}, \mathcal{A}^{n},\left\{P_{\theta}^{n}: \theta \in(\Theta, \mathcal{T}, Q)\right\}\right)
$$

corresponding to a $n$-sized sample of the observed distribution. See the remark below.

(iii) Note that, given $A \in \mathcal{A}$, Fubini's theorem yields

$$
\left(P_{\omega, n}^{*}\right)^{P^{n}}\left(I_{1}^{-1}(A)\right)=\int_{\Theta} P_{\theta}(A) d P_{\omega, n}^{*}(\theta)=\int_{A} \int_{\Theta} p_{\theta}\left(\omega^{\prime}\right) \cdot p_{\omega, n}^{*}(\theta) d Q(\theta) d \mu\left(\omega^{\prime}\right),
$$


where $p_{\omega, n}^{*}$ denotes the posterior density with respect to the prior distribution $Q$. Hence, for $\omega \in \Omega^{n}$, the $\mu$-density of $M_{n}^{*}(\omega, \cdot)$ is

$$
\frac{d M_{n}^{*}(\omega, \cdot)}{d \mu}\left(\omega^{\prime}\right)=\int_{\Theta} p_{\theta}\left(\omega^{\prime}\right) \cdot p_{\omega, n}^{*}(\theta) d Q(\theta)
$$

and this is the Bayes estimator of the sampling density $p_{\theta}$ for the loss function $W_{1}^{\prime}$.

Remark. (A precision on measure-theorethical technicalities in the proofs of the previous results) We detail the proof of Theorem 1.(ii), being that of Theorem 2.(ii) (and even that of the last remark of Section 3) similar. It follows from Theorem 1.(i) that, given $(\omega, \theta) \in \Omega \times \Theta$, and writing

$$
C(\omega, \theta):=\sup _{A \in \mathcal{A}}\left|\left(P_{\omega}^{*}\right)^{P}(A)-P_{\theta}(A)\right|^{2},
$$

we have that, given $n \in \mathbb{N}$, there exists $A_{n}(\omega, \theta) \in \mathcal{A}_{0}$ so that

$$
C(\omega, \theta)-\frac{1}{n} \leq\left|\left(P_{\omega}^{*}\right)^{P}\left(A_{n}(\omega, \theta)\right)-P_{\theta}\left(A_{n}(\omega, \theta)\right)\right|^{2} .
$$

To continue the proof we will use the Ryll-Nardzewski and Kuratowski measurable selection theorem as appears in Bogachev (2007), p. 36.

Keeping the notations of this book, we make $(T, \mathcal{M})=(\Omega \times \Theta, \mathcal{A} \otimes \mathcal{T})$ and $X=\mathcal{A}_{0}$ (the countable field generating $\mathcal{A}$ ). Given $n \in \mathbb{N}$, let us consider the map $S_{n}: \Omega \times \Theta \rightarrow \mathcal{P}(X)$ defined by

$$
S_{n}(\omega, \theta)=\left\{A \in \mathcal{A}_{0}: C(\omega, \theta)-\frac{1}{n} \leq\left|\left(P_{\omega}^{*}\right)^{P}(A)-P_{\theta}(A)\right|^{2}\right\}
$$

We have that $\emptyset \neq S_{n}(\omega, \theta) \subset X$ and $S_{n}(\omega, \theta)$ is closed for the discrete topology on $\mathcal{A}_{0}$. Moreover, given an open set $U \subset \mathcal{A}_{0}$,

$$
\left\{(\omega, \theta): S_{n}(\omega, \theta) \cap U \neq \emptyset\right\} \in \mathcal{A} \otimes \mathcal{T}
$$

because, given $A \in \mathcal{A}_{0}$,

$\left\{(\omega, \theta): S_{n}(\omega, \theta) \ni A\right\}=\left\{(\omega, \theta): C(\omega, \theta)-\left|\left(P_{\omega}^{*}\right)^{P}(A)-P_{\theta}(A)\right|^{2} \leq \frac{1}{n}\right\} \in \mathcal{A} \otimes \mathcal{T}$.

So, according to the measurable selection theorem cited above, there exists a measurable map $s_{n}:(\Omega \times \Theta, \mathcal{A} \otimes \mathcal{T}) \rightarrow\left(A_{0}, \mathcal{P}\left(\mathcal{A}_{0}\right)\right)$ such that $s_{n}(\omega, \theta) \in$ $S_{n}(\omega, \theta)$ for every $(\omega, \theta)$ or, which is the same,

$$
C(\omega, \theta)-\frac{1}{n} \leq\left|\left(P_{\omega}^{*}\right)^{P}\left(s_{n}(\omega, \theta)\right)-P_{\theta}\left(s_{n}(\omega, \theta)\right)\right|^{2} .
$$


It follows that

$$
\begin{aligned}
\int_{\Omega \times \Theta} C(\omega, \theta) d \Pi(\omega, \theta) & \leq \int_{\Omega \times \Theta}\left|\left(P_{\omega}^{*}\right)^{P}\left(s_{n}(\omega, \theta)\right)-P_{\theta}\left(s_{n}(\omega, \theta)\right)\right|^{2} d \Pi(\omega, \theta)+\frac{1}{n} \\
& \leq \int_{\Omega \times \Theta} \sup _{A \in \mathcal{A}}\left|M(\omega, A)-P_{\theta}(A)\right|^{2} d \Pi(\omega, \theta)+\frac{1}{n}
\end{aligned}
$$

which gives the proof as $n$ is arbitrary.

\section{Acknowledgements}

This paper has been supported by the Junta de Extremadura (Spain) under the grant Gr18016.

\section{References:}

- Barra, J.R. (1971) Notions Fondamentales de Statistique Mathématique, Dunod, Paris.

- Bogachev, V.I. (2007), Measure Theory, Vol. II, Springer, Berlin.

- Boldstat, W.M. (2004) Introduction to Bayesian Statistics, Wiley, New Jersey.

- Ferguson, T.S. (1983) Bayesian density estimation by mixtures of normal distributions in Recent advances in statistics", pages 287-302. Academic Press, New York.

- Florens, J.P., Mouchart, M., and Rolin, J.M. (1990) Elements of Bayesian Statistics, Marcel Dekker, New York.

- Geisser, S. (1993) Predictive Inference: An Introduction, Springer Science+ Business Media, Dordrecht.

- Gelman, A., Carlin, J.B., Stern, H.S., Dunson, D.B., Vehtari, A., Rubin, D.B. (2014) Bayesian Data Analysis, 3rd ed., CRC Press.

- Ghosal, S., Vaart, A.v.d. (2017) Fundamentals of Noparametric Bayesian Inference, Cambridge University Press, Cambridge UK.

- Ghosh J.K., Ramamoorthy, R.V. (2003) Bayesian Nonparametrics, Springer, New York. 
- Lijoi, A., Prünster, I. (2010) Models beyond the Dirichlet Process, in "Bayesian Nonparametrics", ed. by Hjort, N.L., Holmes, C., Müller, P., Walker, S.G., Cambridge Series in Statistical and Probabilistic Mathematics, Cambridge.

- Lo, A.Y. (1984) On a class of Bayesian nonparametric estimates. I. Density estimates. Ann. Statist., 12(1):351-357.

- Nogales, A.G. (1998) Estadística Matemática, Servicio de Publicaciones de la Universidad de Extremadura, Cáceres, Spain.

- Rubin, D.B. (1984) Bayesianly justifiable and relevant frequency calculations for the applied statisticians, The Annals of Statistics, 12(4), 1151-1172. 